\title{
Properties of Lattice Ordered Groups and $\Gamma$ - Semi Groups
}

\author{
V. B. Subrahmanyeswara Rao Seetamraju, Anjaneyulu A., and Sridevi S.
}

\begin{abstract}
In this paper we present the properties of lattice ordered groups derived from the properties of partially ordered groups. The notion of $\Gamma$-semi groups was introduced by Sen in 1981. The concept of $\Gamma$ - semigroups is a generalization of the concept of semigroups Many classical notions of semigroups have been extended to $\Gamma$-semigroups, $(S, \Gamma, \leq)$ is called an ordered $\Gamma$-semigroup if $(S, \Gamma)$ is a $\Gamma$-semigroup and $(S, \leq)$ is a partially ordered set such that $a \leq b \Longrightarrow a \gamma c \leq b \gamma c$ and c $\gamma a \leq c \gamma b$ for all $a, b, c \in S$ and $\gamma \in \Gamma$
\end{abstract}

Index Terms-Partially ordered groups, Lattice ordered groups, semi groups, $\Gamma$ semi groups and ordered $\Gamma$ semi groups

\section{INTRODUCTION}

A semi group $\mathrm{S}$ is said to be partially ordered or a partially ordered semigroup if it is associated with a partial ordering $\leq$ which is defined by ' $\mathrm{a} \leq \mathrm{b}$ implies that $\mathrm{xay} \leq \mathrm{xby}$ for all $\mathrm{x}$, $\mathrm{y}$ in $\mathrm{S}$. The natural partial order which is an obvious partial ordering defined by $\mathrm{a} \leq \mathrm{b}$ if and only if $\mathrm{a}=\mathrm{cb}$ for some $\mathrm{c}=\mathrm{c}^{2}$ E S. This natural partial ordering is compatible with multiplication. Some of the basic properties and results were given by Donald B. McAlister and some of the foundational results are due to A.H. Clifford.

Suppose $\mathrm{G}$ is a partially ordered group i.e., $\mathrm{G}$ is a group partially ordered by $\leq$. Now $\mathrm{a} \leq \mathrm{b}$ if and only if $1 \leq \mathrm{a}^{-1} \mathrm{~b}$ or equivalently $\mathrm{a} \leq \mathrm{b}$ if and only if $1 \leq \mathrm{b} \mathrm{a}^{-1}$.

Let $\mathrm{S}$ and $\boldsymbol{\Gamma}$ be non empty sets. If there exists a mapping $\boldsymbol{S} \times \boldsymbol{\Gamma} \times \mathbf{S} \rightarrow \mathbf{S}$, written $(\boldsymbol{a}, \boldsymbol{\gamma}, \boldsymbol{b})$ by $\boldsymbol{a} \boldsymbol{\gamma} \boldsymbol{b}, \mathrm{S}$ is called a $\boldsymbol{\Gamma}-$ semigroup if $\mathrm{S}$ satisfies $(\boldsymbol{a} \boldsymbol{\gamma} \boldsymbol{b}) \boldsymbol{\mu} \boldsymbol{c}=\boldsymbol{a} \boldsymbol{\gamma}(\boldsymbol{b} \boldsymbol{\mu} \boldsymbol{c})$ for all $\boldsymbol{a}, \boldsymbol{b}, \boldsymbol{c} \boldsymbol{\epsilon} \boldsymbol{S}$ and $\boldsymbol{\gamma}, \boldsymbol{\mu} \boldsymbol{\epsilon} \boldsymbol{\Gamma}$. Let $\mathrm{S}$ be an arbitrary semigroup and $\boldsymbol{\Gamma}$ any nonempty set. Define a mapping $\boldsymbol{S} \times \boldsymbol{\Gamma} \times \mathbf{S} \rightarrow \mathbf{S}$ by $\boldsymbol{a} \boldsymbol{\gamma} \boldsymbol{b}=\boldsymbol{a} \boldsymbol{b}$ for all $\boldsymbol{a}, \boldsymbol{b} \boldsymbol{\epsilon}$ and $\boldsymbol{\gamma} \boldsymbol{\epsilon}$. It is easy to see that $\mathrm{S}$ is a $\Gamma$-semigroup. Hence a semigroup can be considered to be a $\boldsymbol{\Gamma}$-semigroup. ( $\mathrm{S}, \leq$ ) is a partially ordered set such that $\boldsymbol{a} \leq \boldsymbol{b} \Rightarrow$ $\boldsymbol{a} \boldsymbol{\gamma} \boldsymbol{c} \leq \boldsymbol{b} \gamma \boldsymbol{c}$ and $\boldsymbol{c} \boldsymbol{\gamma} \boldsymbol{a} \leq \boldsymbol{c} \boldsymbol{\gamma} \boldsymbol{b}$ for all $\boldsymbol{a}, \boldsymbol{b}, \boldsymbol{c} \boldsymbol{\epsilon} \boldsymbol{S}$ and $\boldsymbol{\gamma} \boldsymbol{\epsilon} \Gamma$

\section{Properties of PARTIAL ORDER GROUPS}

Here we consider the set $\mathrm{G}^{+}$consisting of elements exceeding the identity 1 and has the following properties:

1) $\mathrm{G}^{+}$is a submonoid of $\mathrm{G}$

2) $\mathrm{a} \mathrm{G}^{+}=\mathrm{G}^{+}$a for each $a \in G$

3) 1 is the only invertible element of $\mathrm{G}^{+}$

Manuscript received April 15, 2012; revised May 31, 2012.

V. B Subrahmanyeswara Rao Seetamraju is working as Assistant Professor of mathematics and Controller of Examinations at VKR, VNB \& AGK College of Engineering, Gudivada.

A. Anjaneyulu is with the department of Mathematics, VSR \& NVR College, Tenali, A. P.

Sridevi. S is working as Assistant Professor of mathematics at VKR, VNB \& AGK College of Engineering, Gudivada.
Proposition 2.1 Let $\mathrm{G}$ be a partially ordered group and suppose that $a, b \in G$. Then a and $\mathrm{b}$ have a least upper bound $a \vee b$ in $\mathrm{G}$ if and only if they have a greatest lower bound $a \wedge b$. This is only possible only when $\mathrm{a}^{-1}$ and $\mathrm{b}^{-1}$ have a least upper bound. In particular,

$a \wedge b=a(a \vee b)^{-1} b$

$$
\begin{gathered}
a \vee b=a(a \wedge b)^{-1} b \\
a \wedge b=\left(a^{-1} \vee b^{-1}\right)^{-1} \\
a \vee b=\left(a^{-1} \wedge b^{-1}\right)^{-1}
\end{gathered}
$$

Also for any $g \in G$,

$$
\begin{aligned}
& g(a \vee b)=g a \vee g b \\
& (a \vee b) g=a g \vee b g
\end{aligned}
$$

$$
\begin{aligned}
g(a \wedge b)=g a & \wedge g b \\
& (a \wedge b) g=a g \wedge b g
\end{aligned}
$$

Proof. Refer McAlister Lecture notes, University of Lisbon

Corollary 2.2 The following are equivalent for a partially ordered group $\mathrm{G}$.
1) G is a V-semilattice under $\leq$
2) $\mathrm{G}$ is a $\wedge$-semilattice under $\leq$
3) a V 1 exists for each a $\in G$
4) $a \wedge 1$ exists for each $a \in G$
5) $\mathrm{a} V \mathrm{~b}$ exists for each $\mathrm{a}, \mathrm{b} \in \mathrm{G}^{+}$
6) $a \wedge b$ exists for each $a, b \in G^{+}$
7) for each $a, b \in G^{+}$there exists $c \mathrm{C} \mathrm{G}^{+}$such that $\mathrm{G}^{+} \mathrm{a}$ $\cap \mathrm{G}^{+} \mathrm{b}=\mathrm{G}^{+} \mathrm{c}$.

If $\mathrm{G}$ satisfies one of the conditions in the above corollary, we say that $G$ is a lattice ordered group or simply latticed group.

Definition 2.2.1 A lattice $G$ is called a distributive lattice, if for any a,b,c $\in \mathrm{G}$,
(i) $\mathrm{a} \mathrm{V}(\mathrm{b}$
$\wedge \mathrm{c})=(\mathrm{a} V \mathrm{~b}) \wedge$
(a $\mathrm{V} \mathrm{c})$ and
(ii) $\mathrm{a} \wedge$
(b V

Clearly conditions (i) and (ii) are equivalent.

Definition 2.2.2 A lattice $G$ is called a modular lattice, if for any $a, b, c \in G$ such that $a \leq c$ implies $a \mathrm{~V}(\mathrm{~b} \wedge \mathrm{c})=(\mathrm{a} V \mathrm{~b})$ $\wedge \mathrm{c}$.

Theorem 2.2.3

Let $\mathrm{G}$ be a lattice ordered group under a partial order $\leq$, then $\mathrm{G}$ is a modular lattice under $\leq$.

Proof. The proof is obvious, because if $\mathrm{G}$ is a lattice ordered 
group w.r.t a partial order $\leq$, then $\mathrm{G}$ is a distributive lattice under $\leq$ and every distributive lattice is a modular lattice.

Definition 2.2.4

Two elements $a, b$ of a lattice ordered group $G$ are said to be orthogonal if $a \wedge b=1$.

Proposition 2.2.5

Let $\mathrm{G}$ be a lattice ordered group and let $a, b, c \in G$. If $a \wedge b=1$, then $a c \wedge b c=c$.

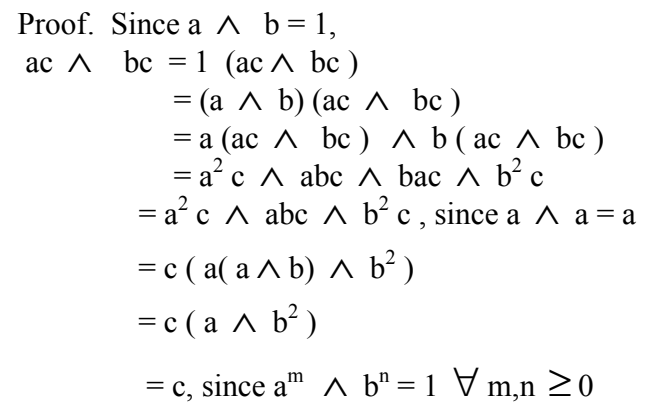

\section{COMPletely Regular $\Gamma$-Semigroups}

An element of a $\Gamma$. Semigroup $\mathrm{S}$ is completely regular if there exists $x \in S$ such that $a=a \alpha x \beta a, a \alpha x=x \beta a$

$\mathrm{A} \Gamma$-semigroup $\mathrm{S}$ is completely regular if all its elements are completely regular

Proposition: If a is an element of a $\Gamma$-semigroup $\mathrm{S}$ and if $a \in\left(S \Gamma a^{2}\right) \cap\left(a^{2} \Gamma S\right)$ then a is contained in the greatest subgroups of $S$ having e as its identity

$$
\begin{aligned}
& \text { Proof: If } a \epsilon\left(S \Gamma a^{2}\right) \cap\left(a^{2} \Gamma S\right), \text { then } \\
& a=x \alpha a^{2}=a^{2} \alpha y \text { for } x, y \in S, \alpha \in \pi \\
& \Rightarrow x \alpha a=x \alpha\left(a^{2} \alpha y\right)=\left(x \alpha a^{2}\right) \alpha y \\
& =\left(a^{2} \alpha y\right) \alpha y\left(\because x \alpha a^{2}=a^{2} \alpha y \alpha\right) \\
& =a \alpha y\left(\because a^{2} \alpha y=a\right) \\
& \therefore x \alpha a=a \alpha y \\
& \text { Let } e=x \alpha a=a \alpha y, \text { e being the identity } \\
& a \alpha e=a^{2} \alpha y=a=x \alpha a^{2}=e \alpha a \\
& e^{2}=(x \alpha a)(a \alpha y)=\left(x \alpha a^{2}\right) \alpha y=a \alpha y=e \\
& \therefore \text { e is idempotent } \\
& \Rightarrow e \epsilon(S \Gamma a) \cap(a \Gamma S)
\end{aligned}
$$

We know $G e=\{a \epsilon S / a \epsilon(e \Gamma S) \cap(S \Gamma e), e \epsilon(a \Gamma S) \cap$ $(S \Gamma a)\}$ is the greatest subgroup having e as its identity

Clearly $a \in G e$

Notation: If a is completely regular element of a $\Gamma$ -semigroups, we denote by $\mathrm{a}^{-1}$, the inverse of $\mathrm{a}$ in the maximal subgroup of $\mathrm{S}$ containing ' $\mathrm{a}$ '

Definition: A $\Gamma$-semigroups $\mathrm{S}$ is left cancellative if $x \alpha a=x \alpha b$ implies $a=b$; for any $a, b, x \in S, \alpha \in \Gamma$ cancellative if it is both left and right cancellative

Definition: $\mathrm{A} \Gamma$-semigroups $\mathrm{S}$ is separative if for any $\mathrm{x}, \mathrm{y}$ $\epsilon S$
1) $x^{2}=x \alpha y$ and $y^{2}=y \alpha x$ imply $x=y$
2) $x^{2}=y \alpha x$ and $y^{2}=x \alpha y$ imply $x=y$

Lemma: In a separative $\Gamma$-semigroup $\mathrm{S}$, for any $x, y, a, b \in S \alpha, \beta \in \Gamma$, the following statements hold
3) $x \alpha a=x \alpha b$ if and only if $a \alpha x=b \alpha x$

4) $x^{2} \alpha a=x^{2} \alpha b$ implies $x \alpha a=x \alpha b$

5) $x \alpha y \beta a=x \alpha y \beta b$ implies $y \alpha x \beta a=y \alpha x \beta b$

6)

Proof:

1)

$$
\begin{aligned}
& \text { If } x \alpha a=x \alpha b \text {, then } \\
& a \alpha(x \alpha a) \alpha x=a \alpha(x \alpha b) \alpha x, \alpha \in \Gamma \\
& \text { And } b \alpha(x \alpha a) \alpha x=b \alpha(x \alpha b) \alpha x \\
& \text { So that }\left(a \alpha x^{2}\right)=(a \alpha x)(b \alpha x) \text { and } \\
& \left(b \alpha x^{2}\right)=(b \alpha x)(a \alpha x)
\end{aligned}
$$$$
\text { By separativity, } a \alpha x=b \alpha x
$$

The opposite implication can be desired easily by using symmetry property

$$
\text { If } x^{2} \alpha a=x^{2} \alpha b \text {, then by part (i) }
$$

$x \alpha a \alpha x=x \alpha b \alpha x$

Hence $(a \alpha x)^{2}=(a \alpha x)(b \alpha x)$ and

$(b \alpha x)^{2}=(b \alpha x)(a \alpha x)$ and thus by separativity $a \alpha x=b \alpha x$

Then by part i), $x \alpha a=x \alpha b$

$$
\begin{aligned}
& \text { Let } x \alpha y \beta a=x \alpha y \beta b, \\
& \text { Then } x \alpha y \beta a \delta y=x \alpha y \beta \delta y \\
& \text { By part i), } y \alpha a \beta y \delta x=y \alpha b \beta y \delta x
\end{aligned}
$$

Multiplying by suitable elements on the right as using part i), we obtain the following equalities:

$$
\begin{aligned}
& (a \alpha y \beta x)^{2}=(b \alpha y \beta x)(a \alpha y \beta x) \\
& (a \alpha y \beta x)(b \alpha y \beta x)=(b \alpha y \beta x)^{2} \\
& \text { Which by separating implies } a \alpha y \beta x=b \alpha y \beta x \\
& \text { But by part i), we have } y \alpha x \beta a=y \alpha x \beta b
\end{aligned}
$$

\section{CONCLUSION}

In this paper, we related semigroups with $\Gamma$-semigroups and we derived different properties $\Gamma$-semigroups by using the properties of semigroups.

\section{ACKNOWLEDGMENT}

Our sincere thanks to Principal, Management and staff of VKR, VNB \& AGK College of Engineering, Gudivada for giving their valuable support in making this work successful.

\section{REFERENCES}

[1] A. H. Clifford and G. B. Preston, Algebraic Theory of Semi groups, American Math. Soc, Providence, vol. 2, no. 7,1967.

[2] M R. Darnel, Theory of Lattice Ordered Groups, Marcel Dekker Inc., New York, N.Y,1995

[3] J. M. Howie, Fundamentals of Semigroup Theory, London Mathematical Society Monographs, New Series 12, Oxford, 1996.

[4] V.V. Bludov and A. M. W. Glass, Conjugacy in lattice-ordered groups and right ordered groups.

[5] M. K, Sen and N. K. Saha, On Г-semigroups I,Bull. Calcutta Math.Soc.78, no. 3,180-186, 1986.

[6] M .Petrich, Introduction to semigroups, Merill, 1973.

[7] S. Bogdanovic, Remarks on $\Gamma$-semigroups and their generalizations- 


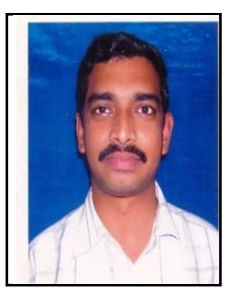

V. B. Subrahmanyeswara Rao Seetamraju completed his M. Sc, from Acharya Nagarjuna University, Guntur in 2000 and worked as Lecturer of mathematics for 6 years at ANR College, Gudivada. Worked as Lecturer of mathematics for 2 years at Dr. BVRICE, Bhimavaram. From August, 2008 he is working as Assistant Professor of mathematics and Controller of Examinations at VKR, VNB \& AGK College of Engineering, Gudivada. He has a total of 12 years of teaching experience.

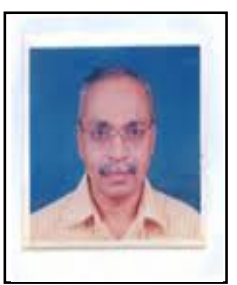

A. Anjaneyulu, Head of the department of Mathematics, VSR \& NVR College, Tenali, A.P., who started writing books for the students of Mathematics, while he was in his research work in 1979. The first book of Dr. A. Anjaneyulu in Mathematics was released in 1983 which was tremendous success. Department of Mathematics is led by Dr A. Anjaneyulu, a renowned teacher, author of 43 text books, books for competitive exams etc. His books on Engineering Mathematics are prescribed by JNTU and all most all degree students of our state use the text books authored by the members of the Department of Mathematics. Sri K Sai Prasad authored 8 text books \& 4 key books. The lecturers of the Department present or retired published 81 books.

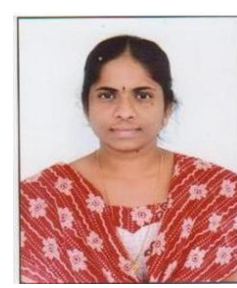

Sridevi S. completed her M.Sc, from Acharya Nagarjuna University in 2004, Guntur and worked as Lecturer of mathematics for 2 years at SCS KALASALA,Gudlavalleru. Worked as Lecturer of mathematics for 2 years at Smt.B.SEETHA POLYTECHNIC ,Bhimavaram. From June, 2008 she is working as Assistant Professor of mathematics at VKR,VNB \& AGK College of Engineering, Gudivada. She has a total of 8 years of teaching experience. 